ISSN: 1130-3743 - ISSN electrónico: 2386-5660

DOI: http://dx.doi.org/10.14201/teoredu2015272115135

\title{
BIOPOLÍTICA Y EDUCACIÓN. MEDICIÓN, ESTANDARIZACIÓN, REGULARIZACIÓN POBLACIONAL
}

\author{
Biopolitcs and education. Measurement, standardization \\ and regularisation of the population
}

\section{Biopolitique et éducation. Mesure, standardisation et régularisation de la population}

\author{
Geo Saura* y Julián Luengo Navas** \\ * Universidad Autónoma de Barcelona. Facultad de Ciencias de la Educación. \\ Campus de la UAB, s/n.08193 Bellaterra (Cerdanyola del Vallès).geo.saura@uab.cat \\ * Universidad de Granada. Facultad de Ciencias de la Educación. Campus \\ Universitario de la Cartuja, s/n. 180171 Granada.jluengo@ugr.es
}

Fecha de recepción: marzo de 2015

Fecha de aceptación: junio de 2015

Biblid [(1130-3743) 27, 2-2015, 115-135]

\section{RESUMEN}

Este texto analiza las pruebas estandarizadas del rendimiento escolar como dispositivo de la biopolítica educativa. Se presenta una breve revisión teórica de la noción de biopolítica, una analítica del poder de organismos economicistas legitimadores de políticas educativas globales y una cartografía sociotécnica de las tecnologías de control docente. El dispositivo de la biopolítica educativa provoca en el alumnado la legitimación de la lógica disciplinaria, la perpetuación de las líneas divisorias (normalidad/anormalidad) y la hegemonía de la estandarización como regularización de los conjuntos poblacionales. Los efectos generados en los docentes se presentan con entrevistas en profundidad de una investigación empírica, destacando dos cualidades: "comparabilidad", cuyos rasgos característicos son cómputo/ 
centro y relación con el todo; y "dividualidad", donde competitividad y sujeción son sus propiedades.

Palabras clave: biopolítica; política educativa; evaluación estandarizada; dispositivo.

SUMMARY

This paper analyzes scholar standardized testing as a dispositif of educational biopolitics. It describes a theoretical review of biopolitics, an analytic of power of the economic agencies that legitimize global education policies and a sociotechnical mapping of teacher control technologies. The dispositif of educational biopolitics causes the legitimizing of disciplinary logic in students, along with the perpetuation of dividing lines (normality/abnormality) and the hegemony of standardization as regularization of population sets. The effects brought about in teachers are presented with in-depth interviews of empirical research, including two qualities: "comparability", of which the characteristics are computing/center and relationship with everything, and "dividuality" where the properties are competitiveness and subjection.

Key words: biopolitics; education policy; standardized testing; dispositif.

\section{SOMMAIRE}

Ce texte analyse les tests standardisés en tant que dispositif biopolitique. Nous présenterons un bref examen théorique de la biopolitique, une analyse des organismes économiques qui légitiment les politiques d'éducation globale et une cartographie socio-technique de technologie de contrôle des professeurs. Le dispositif de biopolitique cause la légitimation de la logique disciplinaire chez les étudiants, la perpétuation des lignes (normale/anormale) et l'hégémonie de la standardisation et de la régularisation. Les effets générés par les enseignants sont représentés par des entrevues détaillées de la recherche empirique, contenant deux caractéristiques: "Comparabilité", dont les caractéristiques sont le calcul/centre et la relation à l'ensemble, et «dividualité» dont les propriétés sont la compétitivité et la sujétion.

Mots clés: biopolitique; politique d'éducation; tests standardisés; dispositif.

\section{INTRODUCCIÓN}

[...] el examen abre dos posibilidades que son correlativas: la constitución del individuo como objeto descriptible, analizable; en modo alguno, sin embargo, para reducirlo a rasgos "específicos" como hacen los naturalistas con los seres vivos, sino para mantenerlo en sus rasgos singulares, en su evolución particular, en sus 
aptitudes o capacidades propias, bajo la mirada de un saber permanente; y de otra parte la constitución de un sistema comparativo que permite la medida de fenómenos globales, la descripción de grupos, la caracterización de hechos colectivos, la estimación de las desviaciones de los individuos unos respecto de otros, y su distribución en una población (Foucault, 1984, 195).

En los discursos contemporáneos, las políticas de evaluación estandarizadas representan el centro de atención internacional, tanto para el diseño de las agendas educativas, como para el análisis de las investigaciones sociológicas, políticas y filosóficas del campo educativo. Los intensos debates sobre los procesos de estandarización y las correlativas reformas educativas que la mayoría de regiones están desarrollando han acaparado las reflexiones y los estudios de la educación en las últimas dos décadas. La intencionalidad de este texto es analizar las políticas de estandarización, extendidas mundialmente, como la representación materializada de un dispositivo de poder y de control de la contemporaneidad biopolítica para ejercer un dominio de los conjuntos poblacionales contenidos en las instituciones escolares. Previamente, sintetizamos algunos interrogantes: ¿Qué se comprende por biopolítica?, ¿desde dónde proceden sus debates?, ¿cómo se investiga la biopolítica en educación?

La noción de biopolítica ha intensificado los debates teóricos en el campo de las ciencias sociales y humanidades en los últimos diez años. Las diversas interpretaciones y traslaciones epistemológicas que se han realizado sobre la biopolítica verifican la existencia de reconstrucciones conceptuales dispares en los diferentes campos de estudios. La introducción de la noción de biopolítica la realiza Rudolf Kjellen a principios del siglo Xx (Esposito, 2004). Kjellen comprende al Estado como "forma viviente», un "conjunto integrado de hombres que se comportan como un único individuo espiritual y corpóreo a la vez» (Esposito, 2004, 28). Unos años más tarde, en 1958, el trabajo de Arendt (2002), aunque no utiliza el término "biopolítica", estudia con un cierto paralelismo al mismo, el modo en el que el gobierno de la modernidad se centra en la producción de la vida biológica. Pero es a partir de los años setenta, debido a los trabajos de Michel Foucault sobre la biopolítica (1987, 2003, 2008, 2009), los que extendieron un interés epistemológico sobre este campo conceptual. A partir de esos trabajos, los debates teóricos sobre el término que nos ocupa no han cesado. La utilización de la biopolítica desde la visión foucaultiana está interrelacionada con la "pletopolítica"; concepto que introduce Rudolf Kjellen para referirse al estudio y a la gestión del cuerpo de la población (Castro, 2011). Para nuestros intereses en el campo de la educación, atendemos principalmente a la biopolítica a partir de la obra de Michel Foucault y otras fuentes que han aludido a la interpretación terminológica del filósofo francés.

Biopolítica es el resultado de la unificación de dos términos: la raíz griega bio, vida; y política, término concerniente a las relaciones de poder generadas y producidas en y desde los gobiernos. La biopolítica es esa práctica de control que los gobiernos, a partir de fines del siglo XVIII, ejercen para gobernar a los individuos introduciendo la capacidad de las estructuras de un "poder de "hacer" vivir y "dejar" 
morir» (Foucault, 2003, 206). La noción de población aparece en ese momento como elemento necesario para controlar y dirigir a un grupo de sujetos por medio de diversos mecanismos de regularización. La tesis mantenida por Agamben (2010, 16) sostiene que la biopolítica es un ejercicio de la soberanía, debido a que «la producción de un cuerpo biopolítico es la aportación original del poder soberano». En la misma fundamentación, Grinberg $(2008,165)$ intenta traspasar la perspectiva de Foucault, argumentando que la actualidad está siendo dominada por un nuevo régimen preocupado en el «dejar vivir y dejar morir». Sloterdijk (2012, 24) se centra en demostrar la formación del homo immunologicus desde la ejecución de las antropotécnicas, "procedimientos de ejercitación, físicos y mentales, con los que los hombres de las culturas más dispares han intentado optimizar su estado inmunológico frente a los vagos riesgos de la vida y las agudas certezas de la muerte». Esta lectura del filósofo alemán es la que Castro-Gómez (2012) lee en "clave Foucault", dividiendo a las antropotécnicas: en biopolitica, como mejora del mundo procedente desde el exterior; y en estética de la existencia, como mejora de uno mismo desde las tecnologías del yo. Esposito (2004) distingue entre una política en nombre de la vida -biopolítica- y una vida sustentada al mando de la política -biopoder-. Además, el término bios no es una designación única de vida natural o física, sino que responde en igualdad de condiciones a la vida cultural (Esposito, 2004).

La biopolítica no es esa forma de poder que actúa directamente sobre los cuerpos de los sujetos para producir individuos dóciles, tal y como era propio el proceder de la anatomopolítica en los regímenes disciplinarios. A la población se la controla ahora de otro modo distinto. Los mecanismos de control ya no van enfocados al cuerpo individual, sino a un cuerpo social (Gallo, 2010). Los individuos están representados (Foucault, 2003, 210) en «... un nuevo cuerpo: cuerpo múltiple, cuerpo de muchas cabezas, si no infinito, al menos necesariamente innumerable. Es la idea de "población"”. La biopolítica tiene que ver con esa idea de población, concebida como medio de interés político, biológico y, por lo tanto, considerada como asunto de poder. Acciones de control poblacional a través de fenómenos de dominio en una duración temporal limitada. La biopolítica utiliza estimaciones estadísticas, medidas globales, regularizaciones y previsiones de amplia magnitud. En ese dominio de la población, no hay una pretensión por controlar al individuo en particular. La intencionalidad de regularización reside ahora en intervenir sobre fenómenos que agrupan a un conjunto amplio de sujetos.

La acogida de los trabajos foucaultianos sobre la biopolítica en el entorno feminista ha propiciado y sigue ejerciendo profundos debates. Los estudios de la corriente feminista han emergido desde los posicionamientos que interrelacionan la noción de biopolítica con los avances sobre las transformaciones del cuerpo, las relaciones de poder y los procesos de subjetividad (Boyer, 2012). Todo ello se produce a través de una pregunta inicial que intenta constatar y dar respuesta para comprender de qué forma los leguajes populares y técnicos construyen los cuerpos y «yoes» biomédicos y biotécnicos (Haraway, 1995). La perspectiva de Preciado 
(2011) reconoce al cuerpo en sí mismo como lugar de opresión que responde a una construcción biopolítica. Incluso la noción misma de género se sustenta tras una producción del biopoder (Preciado, 2008). La biopolítica de Occidente construye la noción «del yo» y «del otro» bajo la distinción entre normal y patológico, en relaciones que confirman cómo «el poder de la biomedicina y de la biotecnología es constantemente reproducido" (Haraway, 1995, 348).

En el campo de la educación, el concepto de biopolítica también está tomando especial interés en la última década, aunque no es así en los trabajos desarrollados en la academia española. Las investigaciones escolares realizadas en otros contextos suelen prestar atención a los dispositivos biopolíticos de las instituciones educativas fundamentándose en las relaciones existentes entre el saber y el poder de las obras de Foucault (1984, 2003, 2008, 2009). Caruso (2005) estudia en el Reino de Baviera la aparición de la enseñanza reguladora como técnica específica de la biopolítica que gobierna a los sujetos por medio del control continuo en los procesos de crecimiento y autorregulación desde finales del siglo XIX. Considerando el desuso de los planteamientos binarios y dicotómicos entre lo normal y lo patológico (Rose, 2007), la escuela funciona bajo la biopolítica, ya que lo excepcional y lo no calculable se ha vuelto parte de lo posible, de una escala de medición (Grinberg, 2011, 15). Las políticas escolares se diseñan bajo formas de mayor control, donde todo queda calculado mediante medición empírica y, a su vez, regido por las nuevas dinámicas de gestión. En la era del management la institución escolar construye sujetos gestionados bajo una lógica de la vigilancia "sindrómica" (Fearnley, 2005) que conlleva gobernar al alumnado entre lo que puede ser prevenido y calculado (Grinberg, 2011). Ball (2013) plantea la necesidad de una genealogía de la clasificación para destacar las formas por las que la educación normativiza y normaliza a los sujetos por medio de la evaluación y la comparación como aparato de disciplina y biopoder. Otra de las investigaciones que abordan la noción de biopolítica en el campo de la educación es el trabajo de Maarten Simons al demostrar que el Espacio Europeo de Educación Superior (EEES) se fundamenta en la biopolítica, debido a los intereses gubernamentales de «economización social", que fomenta un "terror económico" de "bio-economización» enfatizando el aprendizaje como medio de inversión (Simons, 2006, 524). En esta línea que sigue Simons el ejercicio de homogenización que vive Europa, representado por el EEES, utiliza una dimensión biopolítica de los conjuntos poblacionales en la enseñanza superior. De este modo, el EEEs requiere una amplia red de desarrollo global que invierte en la génesis de un tipo de individuo que produzca mejoras económicas en el seno de su territorio. Por ello, uno de los objetivos principales del EEEs es considerar al individuo como "capital humano» con capacidad suficiente para aportar la máxima plusvalía a la institución (Masschelein y Simons, 2002).

\section{LA EVALUACIÓN ESTANDARIZADA COMO DISPOSITIVO DE LA BIOPOLÍTICA EN EDUCACIÓN}

En la actualidad las pruebas de evaluación estandarizadas representan el instrumento esencial del régimen biopolítico en la institución educativa. Los mecanismos 
de control del régimen de la biopolítica no excluyen las técnicas disciplinarias: vigilancia, adiestramiento, jerarquía, inspección u observación directa de los sujetos. Las pruebas de evaluación no son, como señala Foucault (1984) en relación al examen, un elemento de la disciplina y la normalización. Los mecanismos gubernamentales de evaluación y rankings son la extensión de la biopolítica que promueven el ideario neoliberal y la lógica de la performatividad (Veiga-Neto, 2013, 18). Las políticas de estandarización representan a la biopolítica a través de la mensurabilidad de los saberes que controlan y diferencian a los grupos poblacionales. Mediante los resultados de estas pruebas, los conjuntos poblacionales son controlados y gobernados mediante los efectos económicos y políticos que generan. Las estandarizaciones del rendimiento permiten a las agendas educativas globales ejercer ese ejercicio de poder que tiene la capacidad de «designar lo que hace entrar a la vida y sus mecanismos en el dominio de los cálculos explícitos y convierte al poder-saber en un agente de trasformación de la vida humana" (Foucault, 1987, 173).

Los exámenes estandarizados componen la interrelación y legitimación de tres juegos de saber-poder que son inseparables: relaciones de saber; poder disciplinario y normalizador; y biopoder de regulación. La legitimación de las relaciones de saber se perpetúa por medio de los conocimientos específicos que son medidos en las evaluaciones estandarizadas que se encargan de formar un tipo de individuo predeterminado. La medición de esos saberes responde a un poder disciplinario y normalizador que se ejerce directamente en la producción de sujetos dóciles y categorizados en las líneas divisorias de la normalidad y anormalidad. Como elemento concluyente de estas relaciones de saber y poder, el biopoder de regulación se extiende a nivel global por medio de mediciones estandarizadas que introduce a los sujetos en conjuntos poblacionales. Estos tres juegos de saber-poder van aunados, forman parte del mismo aparato.

Las mediciones estandarizadas son el instrumento de control de la institución escolar que opera a través de la individualización, que disciplina y normaliza; y de la totalización, como proceso de control dirigido a la población (Veiga-Neto, 2013). Estos instrumentos de evaluaciones estandarizadas y externas representan el dispositivo de la biopolítica escolar que legitima un saber específico y produce la hibridación de un aparato compuesto por el ejercicio de un poder disciplinario, normalizador y regularizador de los sujetos. Estos dispositivos de normalización contemporáneos de la escuela se vuelven dominantes y dominadores (Veiga-Neto, 2013).

\section{1. ¿Qué es un dispositivo?}

El interés del texto no reside en plantear un debate epistemológico del concepto, aunque es necesario aclarar qué se comprende por un dispositivo, y así poder interpretar las mediciones estandarizadas como un dispositivo biopolítico. El dispositivo es un término que ha sufrido lo que se ha reconocido como "poligénesis conceptual» (Moro, 2013). Esto quiere decir que se trata de un término que 
ha soportado una tergiversación conceptual debida a las múltiples interpretaciones terminológicas que las diversas disciplinas han generado en los diferentes espacios de reflexión teórica. Un dispositivo es:

[...] un conjunto decididamente heterogéneo, que comprende discursos, instituciones, instalaciones arquitectónicas, decisiones reglamentarias, leyes, medidas administrativas, enunciados científicos, proposiciones filosóficas, morales, filantrópicas; en resumen: los elementos del dispositivo pertenecen tanto a lo dicho como a lo no dicho. El dispositivo es la red que puede establecerse entre estos elementos (Foucault, 1991, 128).

Es un "concepto multifacético» (Bailey, 2013) que destaca la primacía de lo fluido, las relaciones de poder productivas y móviles, que interaccionando el saber, los objetos y las prácticas materiales permean, modifican y producen nuevas subjetividades. La noción de dispositivo reúne diversos términos que referencian «a una economía, es decir, a un conjunto de praxis, de saberes, de medidas y de instituciones cuya meta es gestionar, gobernar, controlar y orientar -en un sentido que quiere útil- los comportamientos, los gestos y los pensamientos de los hombres» (Agamben, 2007, 256). Por lo que desvelar los elementos de un dispositivo, alertar sobre su conformación, es lo que Deleuze $(1990,155)$ reconoce como «levantar un mapa, cartografiar, recorrer tierras desconocidas».

Recogiendo la conceptualización foucaultiana del dispositivo, y queriendo traspasar el dualismo analítico, Bailey (2013) desarrolla la diferenciación entre microdispositivos y macrodispositivos para analizar las políticas educativas. El nivel micro comprende las articulaciones de poder material-discursivas que constituyen la expresión material de las relaciones de poder. Los macrodispositivos responden a espacios y lugares en los que se realizan y se disponen las políticas educativas. Los microdispositivos son elementos tácticos y estratégicos enmarcados dentro de una estrategia más amplia -nivel macro- (Bailey, 2013). Todo dispositivo está conformado por la unificación de sus líneas propias: líneas de enunciación, líneas de visibilidad, líneas de enunciación, líneas de fuerza, líneas de objetivación y líneas de subjetivación (Deleuze, 1990). Dicha multiplicidad lineal transforma constantemente la tridimensionalidad distinguida: saber, poder y subjetividad de los tres dispositivos analizados por Foucault. El dispositivo disciplinario (Foucault, 1984) es el que más se centra en la institución escolar y hace referencia a tres procedimientos: la enseñanza, la adquisición de conocimientos del ejercicio pedagógico y la observación recíproca y jerarquizada. El dispositivo de sexualidad (Foucault, 1987) responde al conjunto entramado de relaciones de poder y saber desplegadas respecto al sexo, que describen lo permitido y lo prohibido; lo prescrito y lo ilícito. A partir de estos dos dispositivos -disciplinar y sexual-, aparece el dispositivo de seguridad. El dispositivo de seguridad es esa conjunción de un aparataje mayor que estructura mecanismos para gobernar los conjuntos poblacionales y regular el poder sobre la vida a través de la noción de biopolítica (Foucault, 1987). 


\section{INTERESES Y RELACIONES POR LA LEGITIMACIÓN DE LA ESTANDARIZACIÓN}

Sustentado en la visión de Foucault sobre el poder (1987, 2003, 2008) este apartado no tiene la pretensión de demostrar qué es el poder, sino determinar algunos de sus mecanismos, los efectos surgidos, las relaciones de poder, y en qué ámbitos diferentes se ejercen. Lo que interesa del poder es «saber por dónde pasa la cosa, cómo pasa, entre quiénes, entre qué puntos, de acuerdo con qué procedimientos y con qué efectos» (Foucault, 2008, 14). No hay que cuestionar qué es el poder ni desde dónde viene, sería más acertado plantear cuáles son las formas de ejercerlo, ya que «un ejercicio de poder aparece como un afecto, puesto que la propia fuerza se define por su poder de afectar a otras» (Deleuze, 1987, 100). Es decir, el interés reside en la elaboración de una investigación hacia una analítica del poder, para comprender cómo el poder de la estandarización está siendo legitimado en las instituciones escolares y cuáles son los efectos que están provocando.

La implementación de pruebas de evaluación estandarizadas del rendimiento educativo responde fundamentalmente al movimiento reformista de las políticas educativas globales. El despliegue de estos instrumentos de medición forma parte de una realidad que implica a la mayoría de los sistemas educativos. Es un proceso de cambio que se está consolidando en el tiempo debido a los intereses políticos y económicos que hay detrás de ello. La pujanza ejercida por distintos actores políticos es decisiva para la legitimación de la estandarización a nivel global. Algunos actores como el Fondo Monetario Internacional, el Banco Mundial, la Organización Mundial del Comercio y la Organización para la Cooperación y el Desarrollo Económico (OCDE, a partir de este momento) influyen severamente en las agendas educativas reformuladas en la mayoría de zonas geográficas (Robertson y Dale, 2009). Algunas de las mediciones internacionales que han propagado su extensión por la mayoría de contextos globales han sido PISA (Programme for International Student Assessment) de la $\mathrm{OCDE}^{1}$, junto a TIMSs (Trends in International Mathematics and Science Study) y PIRLS ${ }^{2}$ (Progress in International Reading Literacy Study), pruebas de evaluación de la Asociación Internacional para la Evaluación del Rendimiento Educativo.

De todos ellos, la OCDE mediante PISA ha legitimado su poder ya que la expansión y consolidación de estas pruebas es una realidad en la mayoría de sistemas escolares. Esta organización ha terminado convirtiéndose en el dirigente principal de la gobernanza educativa global (Meyer y Benavot, 2013). La hegemonía de la OCDE se ha llevado a cabo a través de su reputación, los buenos argumentos y la persuasión a los países miembros y agregados (Jakobi y Martens, 2007). La legitimación de sus relaciones de poder y de control las ejercita diagnosticando,

1. Para acceder a la información expuesta por la OCDE sobre PISA, ver, http://www.oecd.org/ pisa/aboutpisa/.

2. Información sobre TIMSS y PIRLS: http://www.iea.nl/timss_2015.html; http://www.iea.nl/ pirls_2016.html. 
enjuiciando, asesorando e influyendo en la creación de nuevas políticas educativas que se extienden globalmente. La generación de ideas, la evaluación de las políticas y la producción de los datos agrupan a todo el conjunto de intereses que conforman los mecanismos de gobernanza de la OCDE (Martens y Jakobi, 2010). Todos los argumentos anteriormente descritos son los principales motivos que impulsan a los países participantes a promover políticas de estandarización en sus sistemas educativos.

Estas mediciones, que crean y consolidan estándares educativos, se hegemonizan como el principio básico para optimizar los sistemas educativos a nivel mundial. Son mecanismos globales para controlar a los países bajo el equilibrio y la regularidad. De este modo, la educación está sujeta al poder de la regularización, lo que, en el caso de PISA, representa la gran regulación global de la población educativa que ha proliferado desde comienzos del siglo xxI. Estas pruebas, como dispositivo biopolítico, no han excluido a los mecanismos de poder disciplinarios. El examen sigue perpetuándose como medio punitivo dirigido hacia los sujetos y como guía del aparato disciplinario del régimen escolar. Los exámenes de PISA unifican la docilidad de la disciplina con la regularización de la biopolítica. Los estándares se presentan como estrategias para mejorar la educación, pero lo que realmente genera es un nuevo modo de gobernanza de los países regida a través de un proceso de estandarización que los compara. La OCDE, con sus funciones de gobernanza epistemológicas e infraestructurales, se perpetúa así a nivel global formulando políticas que crean países y crean personas (Sellar y Lingard, 2013).

¿Por qué se han legitimado estas pruebas de evaluación? La respuesta puede ser dada por medio de otra cuestión: «Puede el análisis del poder o los poderes deducirse, de una manera u otra, de la economía?» (Foucault, 2003, 22). La legitimación de los procesos de estandarización del rendimiento, aunque no únicamente, tiene mucho que ver con la perpetuación del economicismo como poder de dominación de los sistemas educativos. PISA es considerada como medida internacional comparativa del capital humano de las regiones, utilizada como indicador del potencial de competitividad económica mundial (Rizvi y Lingard, 2009). Es un modo para comprender a la educación como proceso competitivo y medio de crecimiento económico. Bajo esta racionalidad economicista, Hanushek y Woessmann (2011) interrelacionan los resultados de las pruebas PISA y el crecimiento económico de los países. Por medio del informe anual Education at a Glance, de los indicadores de la OCDE, se promueve un discurso hegemónico que justifica la necesidad de la evaluación cuantificada, estandarizada y comparativa como medio para la mejora económica y social. Estos principios han llevado a considerar a PISA como un aparato que garantiza la «economización de la política educativa" y la "educacionalización de la política económica» (Sellar y Lingard, 2013, 191). De este modo, los ejercicios del dispositivo de biopoder escolar tienen su finalidad en la economía y en el aumento de las relaciones de producción. Todo ello se ha concretado en reformas educativas que recorren la mayoría de sistemas educativos para perpetuar las pruebas de evaluación 
en los centros educativos. Con el escenario creado de lucha económica entre regiones y países a escala global, se establece una transformación ontológica de los planteamientos generales de la educación. Todo esto ha llevado a originar nuevas políticas educativas para adaptarse a los procesos que evalúan, comparan, clasifican y ordenan las actuaciones en los diferentes países (Sahlberg, 2006). Para que ello se haga efectivo, se introducen nuevas tecnologías de control y poder que modifican el modo de proceder en la institución escolar.

\section{CARTOGRAFíA SOCIOTÉCNICA}

Las agendas educativas de estas promulgaciones globales que formalizan el dispositivo de la biopolítica educativa están fundamentadas en la incorporación de diversos mecanismos, técnicas y tecnologías de poder/control que gobiernan y dirigen a los conjuntos poblacionales. Es todo un entramado normativo que promueve incesantemente un ejercicio clasificador dirigido al "hombre-especie» conformado por un amplio aparataje de estrategias de regulación y mecanismos de control de los individuos (Foucault, 2003). El análisis de las partes que conforman este dispositivo biopolítico trata de referenciar las herramientas que desarrollan una regulación de los conjuntos poblacionales y las acciones que dirigen los procesos educativos hacia la máxima optimización de los resultados obtenidos en la medición de estándares. Son herramientas e instrumentos que controlan las acciones del profesorado con la intención de dirigir la praxis para aumentar cuantitativamente el rendimiento escolar. Entre estas tecnologías de poder/control se pueden mencionar las pruebas de evaluaciones estandarizadas y externas, la publicación del rendimiento escolar por medio de tablas o rankings, los sistemas de incentivos económicos en los docentes, la rendición de cuentas, las nuevas formas de gerencialismo y las agencias de evaluación que exigen que el profesorado justifique las actuaciones de su praxis. Esta descripción sobre estas políticas educativas de poder/control es lo que Deleuze (1995) sostiene como la necesidad de acometer una cartografía sociotécnica capaz de describir los mecanismos de vigilancia poblacional propios de la sociedad de control. Este análisis está conformado por mecanismos que controlan la función docente, dando lugar a nuevos modos de actuar y comprender el rol de la escuela pública. Estos cambios vienen presenciados por la materialización de políticas educativas con carácter global que sugieren a la enseñanza pública la adopción de principios de mercado, planteamientos éticos propios del ámbito privado y formas de actuación normalizadas en el sector empresarial. Todas estas actuaciones propician la implantación a nivel internacional de dinámicas de privatización encubierta (Ball y Youdell, 2008; Burch, 2009) que están logrando un movimiento de reformas educativas globales con carácter gerencial (Verger y Altinyelken, 2012). 


\section{EFECTOS/AFECTOS}

¿Cuáles son los efectos producidos por los dispositivos de biopolítica directamente en los seres inmersos en la institución educativa? Las consecuencias son diversas dependiendo del interés y la atención prestada. El interés aquí recae en demostrar cómo este biopoder desplegado en los procesos de regularización extensivos globalmente guía a los sistemas educativos, genera procesos de exclusión, legitima nuevas formas de control y construye nuevos sujetos sociales. Lo que es innegable son «los diferentes modos de subjetivización del ser humano en nuestra cultura [...] los tres modos de objetivización que transforman a los seres humanos en sujetos" (Foucault, 1988, 227), comprendidos en sujetos productivos, prácticas divisorias y los modos en que se convierten en sí mismos. A este respecto, Tassin (2012) establece esta triangulación de las etapas foucaultianas diferenciando entre: 1) objetivación científica del sujeto; 2) objetivación experiencial de un sujeto atrapado en divisiones normativas; 3) objetivación del sujeto como sujeto. En sí mismo, el significado que se otorga al término "sujeto" hace referencia tanto a la dependencia y sometimiento de unos individuos respecto a otros, así como a un sujeto atado a su propia identidad debido a la conciencia o al conocimiento de sí mismo (Foucault, 1988). Recogiendo parte de este planteamiento foucaultiano, Butler $(2010,12)$ define la sujeción como «el proceso de devenir subordinado al poder, así como el proceso de devenir sujeto".

El análisis de los efectos del dispositivo biopolítico de la medición estandarizada en los sujetos se realiza en una diferenciación estructural distinguida entre alumnado y profesorado. En relación al alumnado, interesa demostrar, mediante la reflexión y la analítica, cómo la medición estandarizada se encarga de producir sujetos bajo procesos de disciplinarización, normalización y regularización. Desenmarañar las líneas que formalizan el dispositivo biopolítico de la estandarización para cartografiar los procesos de objetivación científica y experiencial del sujeto alumnado. El análisis de la transformación del sujeto docente se realiza por medio de la exposición de extractos de entrevistas en profundidad recogidos en un proceso de investigación empírica. Los efectos generados en el profesorado se deben a que las transformaciones vertiginosas que los sistemas escolares están adoptando, donde la estandarización es el epicentro, están provocando que los profesores estén constantemente cuestionándose quiénes son y qué quieren hacer (Simons y Masschelein, 2008).

\section{Alumnado}

El biopoder de las pruebas de evaluación estandarizadas está constituido por la intersección del poder, disciplinario, normalizador y regularizador. El efecto que produce la estandarización en el alumnado es ejercido por dicha interrelación. Los dos primeros ejercicios de poder, "disciplinario" y "normalizador", constituyen la esencia misma de la institución escolar como órgano legitimado de control social. El examen, instrumento representativo del ritual de los regímenes punitivos 
(Foucault, 1984), se ejerce como medio de producción de la docilidad, la disciplina y la normalización. Las pruebas de evaluación estandarizadas se legitiman como dispositivos de poder que objetivan al sujeto por medio de la línea divisoria que enjuicia entre normalidad y anormalidad. Representa lo permitido y lo prohibido, a la vez que categoriza a los sujetos dentro de esa "operación de normalización" (Lazzarato, 2005). Es ese dispositivo encargado de «trazar el límite que habrá de definir la diferencia respecto de todas las diferencias, la frontera exterior de lo anormal» (Foucault, 1984, 188). Representan a una "reacción negativa" del poder, como "tecnología del poder que expulsa, excluye, prohíbe, margina y reprime» (Foucault, 2001,53 ) los conocimientos que poseen otros grupos, como a los propios sujetos que no se ajustan a la "normalización». En cuanto a la "reacción positiva", se apunta a esos mecanismos de poder que fabrican sujetos normalizados, un poder que observa y se multiplica a partir de sus propios efectos. Así, las evaluaciones se legitiman como la "normalidad" a la que todos los sujetos deben adecuarse. Los sujetos que no se ajustan a esa norma preestablecida, desde las directrices de los organismos economicistas que dominan la educación mundial, quedan excluidos en esos límites divisorios otorgados por la imposición de la verdad.

El elemento que va a circular de lo disciplinario a lo regularizador, que va a aplicarse del mismo modo al cuerpo y a la población, que permite a la vez controlar el orden disciplinario del cuerpo y los acontecimientos aleatorios de una multiplicidad biológica, el elemento que circula de uno a la otra, es la norma (Foucault, 2003, 217).

El tercer tipo de poder ejercido con el dispositivo de la estandarización, el poder de la "regularización", se lleva a cabo tras los efectos del poder disciplinario y del normalizador. Se trata de la creación de los grupos poblacionales en la institución escolar, tras ese "medir en términos cuantitativos y jerarquizar en términos de valorar las capacidades, el nivel, la "naturaleza" de los individuos" (Foucault, 1984, 188). El poder de regularización necesita la noción de población como medio para gobernar a los sujetos bajo el agrupamiento de conjuntos poblacionales definidos. La población evaluada de cada centro escolar, región y país representa a un total mensurable y comparable en dimensión global que ejerce poder sobre la vida del alumnado para gobernarlo.

Por medio de los tres poderes que se entrecruzan en el dispositivo biopolítico de la estandarización (disciplinario, normalizador y regularizador) se constituye un «sistema comparativo que permite la medida de fenómenos globales, la descripción de grupos, la caracterización de hechos colectivos, la estimación de las desviaciones de los individuos unos respecto de otros, y su distribución en una población» (Foucault, 1984, 195). Este dispositivo se sustenta en la estadística (Lazzarato, 2005), como medio indispensable de los instrumentos de seguridad que los gobiernos necesitan para gobernar a la población. El poder de regularización, fenómeno propio del proceso de globalización, es, a su vez, causa y efecto de unión y división social (Bauman, 2010). El biopoder ejercido a través de las pruebas de evaluación hegemoniza los conocimientos evaluables. Es un modo de establecer 
la objetivación científica del sujeto, la legitimación de los saberes específicos que normalizan los conocimientos y, a la vez, excluyente de los restantes saberes no evaluables. Con estas mediciones se posibilita a los sujetos una comprensión de ellos mismos, un instrumento de poder que genera sujetos determinados por lo medible. La evaluación es en sí una actividad de poder que compone sujetos, forjada y dependiente en unos valores concretos, y órgano influyente directamente en lo que se aprende y en las formas de llevar a cabo el aprendizaje (Stobart, 2008). Desde esta concepción, las pruebas de evaluación se manifiestan como un «arte de gobernar» a la población escolar. Dispositivo de poder disciplinario, normalizador y regularizador que está construyendo nuevos sujetos sociales.

\section{DOCENTES}

Este apartado expone algunas entrevistas en profundidad que, siguiendo la línea de trabajos previos (Ball, 2003; Ball 2009), intenta comprender los efectos que la implementación de las políticas educativas genera en el profesorado. Es una justificación analítica por medio de los resultados de un proceso de investigación empírica, para demostrar cómo el dispositivo biopolítico de la evaluación estandarizada está generando nuevos sujetos docentes. El biopoder de la estandarización ejercido directamente sobre el profesorado y el espacio escolar a través de las tecnologías de poder/control genera múltiples efectos. Destaca, entre ellos, la construcción de sujetos docentes a través de la aparición de dos nuevas cualidades: «comparabilidad» $\mathrm{y}$ «dividualidad».

\subsection{Comparabilidad}

La comparabilidad es una de las cualidades que aparecen en la docencia como consecuencia de la implementación de las mediciones estandarizadas. Los rasgos que la caracterizan son: el cómputo/centro y la relación con el todo.

El cómputo/centro hace referencia al proceso por el cual el centro escolar se considera como la unidad mínima de medición del rendimiento educativo. Ya no importa el resultado del alumno, ni del aula, ahora lo esencial es el resultado total que cada institución pueda lograr. Así lo relatan dos participantes ${ }^{3}$ :

Yo tengo que dar todos los contenidos, unas veces unos los pillan mejor, otras peor, pero necesito que todos por lo menos lo hayan oído alguna vez todo. Antes podías dejarte los últimos temas sin dar, pero ahora, eso es imposible, porque las pruebas de evaluación miden todo lo que hay en el libro. Mari Carmen.

3. El proceso metodológico de los extractos que se exponen sigue los planteamientos de un proyecto de investigación más amplio. Para atender las fases de la investigación empírica, ver artículo de los autores 2013). 
Ahora los tiempos son otros, yo tengo que dar todo para que vaya preparados a las pruebas de evaluación. Antes me podía dedicar a los alumnos en sí, sin mirar los tiempos, pero ahora, antes de mayo todo tiene que estar dado, necesitamos el mejor resultado posible dentro de las posibilidades en esta zona. Francisco.

El alumnado como sujeto sustancial se desvanece, debido a que la relevancia que el proceso educativo le otorga se inscribe según su valía para mejorar el cómputo total de la institución. Una de las entrevistadas revela que el día que se van llevar a cabo las pruebas de evaluación estandarizadas no "interesa" que acuda al centro el alumnado con más atraso académico. Es una de las estrategias que el centro educativo busca con la finalidad de que el resultado total sea lo más positivo posible.

Yo tuve la suerte, de que dos niños que hubieran bajado mucho el nivel de las notas, que eran dos niños [...] uno se fue el mes antes y el otro se rompió la muñeca, por esa casualidad tuve mejor nota, porque si esos dos hubieran venido, hubiera tenido peor nota. Mari Carmen.

Una de las directoras, orientada por el afán de obtener los mejores resultados del centro y de evitar la reducción de la plantilla docente, expresa la necesidad de que repitan curso dos alumnos. Los niños forman parte del juego competitivo que genera la optimización permanente de resultados y recursos.

Tengo que hacer que en $2 .^{\circ}$ de primaria repitan dos niños. Son alumnos que tienen malos resultados. Si no repiten me quitan un profesor del centro, yo tengo que calcular para no perder plantilla, tengo que pensar en mi centro y en mis profesores. [...] Soy una líder pedagógica. Rosa.

El cómputo/centro es una esencia de la comparabilidad que se generaliza por las distintas políticas escolares. Representa diversas estrategias con capacidad para construir a los sujetos docentes por medio del poder de la regularización comparativa entre centros, localidades, regiones y países. A su vez, es un medio de control y de rendición de cuentas del trabajo docente, que computa los tiempos y la programación de las acciones del profesorado y alumnado.

Por medio de la plataforma digital tenemos que estar subiendo constantemente todo lo que hacemos en el centro. Ahora ya no es control por el papel, ahora son "subida de datos» constantemente, yo me siento más controlada, porque tienen que saber todo lo que yo hago, con cifras y tiempos establecidos. Sandra.

La comparabilidad se vale de otro de sus rasgos distintivos: la relación con el todo. Con la pretensión de que el trabajo del profesorado repercuta en el cómputo mensurable de la institución, la praxis docente se encamina hacia un todo más amplio que supera el aula como elemento mínimo de medición. De ese modo se instala en el interior del profesorado la concepción de que su práctica profesional debe centrarse en aquellas actuaciones que contribuyan al logro del cómputo 
máximo mensurable. Por ello la función del docente ya no se vincula al alumnado ni el aula, sino que las sobrepasa como unidades de medida. La unidad mínima de medición en estos momentos es el centro. De este modo lo expresa uno de los directores participantes:

Los maestros tienen que pensar en los mejores resultados para el colegio. Claro que me preocupo por los resultados. Al final de curso, en la evaluación, yo les decía: interesa que los resultados sean buenos. Antonio.

Los sujetos han hecho suya la identidad-centro, se reconocen como un eslabón primordial y necesario para que la institución escolar pueda competir por los mejores resultados académicos. El profesorado, al formar parte del todo -el centro como resultado mensurable-, asimila la comparabilidad como un rasgo esencial que otorga valía a su profesión.

Así lo expresa un director de un centro situado en una zona de vulnerabilidad social y económica.

Pues claro que lo importa al final es el centro, eso es así. Hay quienes cogen mejor las cosas y quienes no, pero al final, lo que importa es que nuestra escuela sea mejor o peor en relación a nuestros semejantes, no en comparación con escuelas del centro, eso es diferente. Por lo menos que en lo básico seamos buenos. De esa forma te sientes orgulloso de tu trabajo, si el total evaluado es bueno o no lo es. Francisco.

El cómputo total del centro ejerce un poder que controla y gobierna la forma de pensar la educación y las relaciones escolares. La inspección educativa se puede considerar, en este contexto de mediatización e influencia externa, como un vector que intensifica y concreta todos los aspectos técnico-pedagógicos que se deben desarrollar en el aula.

La inspección me controla, esa es su función. A todos nos interesa sacar buenos resultados en las pruebas de evaluación, porque hay una relación directa con los resultados PISA. A mí me interesa que la inspección vea que las cosas se hacen bien aquí, y al inspector también le interesa que sus centros tengan buenos resultados, y Andalucía quiere buenos resultados respecto a otras comunidades de España, y al país con Europa, eso es así, esto es una cadena, nadie puede fallar. Laura.

¿Dirección en relación a la inspección? Yo como director, me preocupo que mi centro tenga buenos resultados, lo que me interesa es un resultado global, que luego la inspección pueda valorarme como positivo, que pueda tener buenos resultados en comparación con los otros centros. Francisco.

Siguiendo esa cadena de transmisión de poder y control, como eslabón previo a la inspección, la dirección escolar se consolida como la figura que asume la responsabilidad de asegurar la identidad-centro. La dirección escolar orienta sus criterios de actuación para transformar a los docentes, y que éstos integren en su 
identidad profesional el valor que otorga la optimación de las pruebas estandarizadas que afectan al centro como un todo mensurable.

Claro que tenemos presiones desde arriba, a mí la directora me dice que tenemos que sacar buenos resultados, porque nuestro centro tiene que quedar bien al final de todo. Sandra.

\subsection{Dividualidad}

La dividualidad es otra cualidad que se origina desde el biopoder como consecuencia de la legitimación de las pruebas de evaluación estandarizadas en las instituciones escolares. En estos momentos, "no estamos ante el dualismo "individualismo-masa". Los individuos han devenido "dividuales" y las masas se han convertido en indicadores, datos, mercados o bancos" (Deleuze, 1995, 281). Por ello, las políticas educativas de estos tiempos no se interesan por los seres independientes e individuales. Se trata de generar seres dividuales que dependan unos de otros. En el caso del profesorado, docentes que forman parte del dividendo institucional. Divisores activos que produzcan el máximo cociente o resultado para el centro educativo como unidad mínima de valor. La dividualidad está conformada por dos rasgos: la competitividad y la sujeción que, en un primer momento, podrían parecer términos antagónicos. Sin embargo, competitividad y sujeción son términos que se necesitan uno del otro para responder con fidelidad y eficacia a las diversas formas de control ejercidas en el cuerpo docente.

La competitividad es una de las características esenciales de estas reformas educativas que se han instalado con fuerza en las prácticas y en los procesos escolares. Los dividuos de las instituciones educativas están instalados en ambientes sometidos a incesantes acciones de lucha. Una estrategia política que ha introducido estos componentes competitivos en los ambientes escolares ha consistido en la implementación de sistemas de incentivos económicos al profesorado como recompensa por el «trabajo bien hecho». Con ello nos referimos a prácticas extendidas globalmente, fundamentadas en la incorporación de incentivos económicos dependiendo del rendimiento académico de los escolares. En el panorama español, como han señalado críticamente, una política de incentivos económicos en los docentes regida principalmente por la evaluación de estándares comenzó a desarrollarse desde 2008 en Andalucía (Luengo y Saura, 2012; Merchán, 2012). Se trata de una herramienta de control sobre los sujetos que, a través de la competitividad, incentiva a los docentes para que su desempeño se focalice hacia las tareas escolares evaluables y que otorgan la recompensa económica. Una de las participantes del proceso empírico comenta este estado de competitividad que se está originando por medio de la estandarización.

Puede ser que ahora la valía sean los resultados en las pruebas, sí. Quizá antes era más la experiencia, ahora el que vale como maestro, se ve claramente aquí, y a todos nos interesa que sean buenos los resultado. Elena. 
El ambiente competitivo que se instala en las escuelas genera en los docentes la necesidad de producir mayores resultados. Este proceso se puede considerar como una modalidad de control en la que los docentes, sujetados unos a otros, se miran, se observan e intentan superarse en un proceso de emulación constante.

Claro que quiero sacar mejores calificaciones que mi compañera, porque si tenemos una clase que más o menos es la misma, porque los niños son de la misma zona, pues yo supongo que se ve quién es mejor maestra si hay diferencia en los resultados de estas pruebas. Laura.

Además de la competitividad, los seres dividuales necesitan estar en constantes procesos de sujeción entre ellos para formalizar la totalidad numérica de la institución. Los distintos actores del centro, como sujetos dependientes entre sí, se necesitan. La evaluación estandarizada cumple esta función al sujetar unos a otros.

Ahora trabajamos todos juntos, tenemos más reuniones de ciclo, tu trabajo no era como antes, encerrada en el aula. El director nos promueve que nos reunamos más a menudo, sobre todo al final de curso, cuando están cerca las pruebas de evaluación de diagnóstico. Rosa.

Todo sujeto está conformado por un poder que le precede y, a la misma vez, ese poder es efectuado por el propio sujeto. El sujeto emerge desde una simultaneidad caracterizada por el efecto de un poder anterior a él, y como condición de posibilidad de potencia radicalmente condicionada (Butler, 2010). El profesorado está subordinado al poder normativo legitimado por las evaluaciones estandarizadas. A su vez, ese poder es efectuado por el profesorado, al conformarse como sujeto evaluador que viene otorgado por el poder de la medición de estándares.

Le damos mucha importancia a las evaluaciones de diagnóstico, somos el centro al completo. Al aceptar el Programa de Calidad, nos hemos comprometido a ello. Yo personalmente, dedico todos los viernes a la evaluación de lengua y matemáticas. Si quieres sacar buenos resultados en las evaluaciones, tienes que evaluar por competencias y adaptarte a ellas. Sandra.

El efecto que se origina mediante un dispositivo es la producción de sujetos sujetados bajo determinados efectos de saber/poder (García Fanlo, 2011). El dispositivo de la estandarización origina docentes sujetados bajo este tipo de relaciones, las cuales son intrínsecas a los procesos hegemónicos de la estandarización del rendimiento de los escolares. 


\section{CONCLUYENDO POR MEDIO DE ALGUNAS CONSIDERACIONES}

La promulgación de políticas educativas fundamentadas en prácticas de control y regulación poblacional en los centros escolares es una realidad extendida a nivel global. Este tipo de acciones responden a los fundamentos epistemológicos del régimen de la biopolítica (Foucault, 2001), con las características propias de la sociedad de control (Deleuze, 1995). En las instituciones educativas, como espacio delimitado para aplicar procesos de poder y control sobre la población, las pruebas de evaluación estandarizadas del rendimiento escolar constituyen el dispositivo principal de la biopolítica educativa. Las diversas políticas de evaluación estandarizada que se extienden por los sistemas educativos tienen su origen en intereses de organismos economicistas que se han convertido en actores políticos hegemónicos en la toma de decisiones. El examen continúa representando el instrumento esencial para producir el régimen de docilidad, disciplina y regularización de los centros. En estas actuaciones del sistema de control, la OCDE, a través de las pruebas PISA, ha llegado a consolidarse como el órgano más representativo en la nueva gobernanza educativa global. Además de la estadística y las mediciones globales, el dispositivo de biopolítica escolar está acompañado de nuevas tecnologías de poder/control que, asemejando las acciones escolares al ámbito privado, modifica el modo de proceder en la escuela pública. Todas estas tecnologías se apoyan en una racionalidad economicista que Lyotard (1991) comprende como una relación performativa de optimización de la relación input/output. Los efectos que el dispositivo de biopolítica educativa está provocando son diversos, pero lo que es innegable es la conformación de nuevos sujetos sociales. En el caso del alumnado, el dispositivo de la biopolítica educativa provoca la legitimación de la lógica disciplinaria, la perpetuación de las líneas divisorias (normalidad/ anormalidad) y la hegemonía de la estandarización como regularización de los conjuntos poblacionales. En los discursos políticos y jurídicos de la modernidad prevalece una estructura de poder y verdad "deshumanizadora", en el sentido de que obstaculiza la construcción positiva del sujeto (Santos, 2013). El poder sobre la vida del alumnado se produce a través de la unificación representativa de la disciplina como "anatomopolítica del cuerpo humano» y los controles reguladores como «biopolítica de la población» (Foucault, 1987, 168). Por su parte, los efectos generados en los docentes son múltiples dependiendo de los intereses analíticos. En este caso, por medio de algunos relatos recogidos tras una investigación empírica, se ha atendido al proceso por el cual el dispositivo de la biopolítica está incidiendo severamente en la identidad profesional del profesorado. Las diversas actuaciones de control, propias del régimen de la biopolítica, generan en los sujetos estados de inseguridad, desigualdad e individualización (Lazzarato, 2009). Estos procesos están construyendo nuevos sujetos sociales, caracterizados por la emersión de dos cualidades: "comparabilidad", cuyos rasgos son el cómputo/ centro y la relación con el todo; y "dividualidad", siendo la competitividad y la sujeción sus atributos. 


\section{REFERENCIAS BIBLIOGRÁFICAS}

Agamben, G. (2007) ¿Qué es un dispositivo? Sociológica, 73, 249-264.

Agamben, G. (2010) Homo sacer. El poder soberano y la nuda vida. Valencia, Pre-textos.

ARENDT, H. (2002) La condición humana. Barcelona, Paidós.

BAiley, P. L. J. (2013) The policy dispositif: historical formation and method. Journal of Education Policy, 28 (6), 807-827. http://dx.doi.org/10.1080/02680939.2013.782512.

Ball, S. J. (2003) The Teacher's Soul and the Terrors of Performativity. Journal of Education Policy, 18 (2), 215-228. http://dx.doi.org/10.1080/0268093022000043065.

BALL, S. J. (2009) Education reform, teacher professionalism and the end of authenticity, en Simons, M.; Olssen, M. y Peters, M. A. (eds.) Re-Reading Education Policies. Rotterdam, Sense Publishers, 667-682.

BalL, S. J. (2013) Foucault, power and education. New York and London, Routledge.

Ball, S. J. y Youdell, D. (2007) Privatización encubierta en la educación. Brussels, Education International.

Bauman, Z. (2010) La globalización. Consecuencias humanas. México D. F., Fondo de Cultura Económica.

Boyer, A. (2012) Biopolítica y filosofía feminista. Revista de Estudios Sociales, 43, 131-138. http://dx.doi.org/10.7440/res43.2012.11.

Burch, P. (2009) Hidden markets. The new education privatization. New York and London, Routledge.

Butler, J. (2010) Mecanismos psíquicos del poder. Teorías sobre la sujeción. Madrid, Cátedra.

Caruso, M. (2005) La biopolítica en las aulas. Prácticas de conducción en las escuelas elementales del Reino de Baviera, Alemania (1869-1919). Buenos Aires, Prometeo.

CAStro, E. (2011) Biopolítica: orígenes y derivas de un concepto, en CASTRO, E.; GrinBerg, S.; O’Malley, P. y Veiga-Neto, A. (eds.) Biopolítica, gubernamentalidad, educación, seguridad. Buenos Aires, UNIPE, 5-11.

Castro-Gómez, S. (2012) Sobre el concepto de antropotécnica en Peter Sloterdijk. Revista de Estudios Sociales, 43, 63-73. http://dx.doi.org/10.7440/res43.2012.06.

Deleuze, G. (1987) Foucault. Barcelona, Paidós.

Deleuze, G. (1990) ¿Qué es un dispositivo?, en Balbier, E.; Deleuze, G. y Hubert, L. (eds.) Michel Foucault filósofo. Barcelona, Gedisa, 155-163.

Deleuze, G. (1995) Post-scriptum sobre las sociedades de control, en Deleuze, G. (ed.) Conversaciones. Valencia, Pre-textos, 277-286.

Esposito, R. (2004) Bíos, biopolítica y filosofía. Buenos Aires, Amorrortu.

FEARnley, L. (2005) "From Chaos to Controlled Disorder": Syndromic Surveillance, Bioweapons, and the Pathological Future. ARC Working Paper, 25, 1-42.

Foucault, M. (1984) Vigilar y castigar: nacimiento de la prisión. México D. F., Siglo XXI.

Foucault, M. (1987) Historia de la sexualidad. 1. La voluntad del saber. Madrid, Siglo XXI.

Foucault, M. (1988) Por qué estudiar el poder: la cuestión del sujeto, en DreYFus, H. L. y Rabinow, P. (eds.) Michel Foucault: más allá del estructuralismo y la hermenéutica. México D. F., Universidad Nacional Autónoma de México, 227-234.

Foucault, M. (1991) El juego de Michel Foucault, en Foucault, M. (ed.) Saber y verdad. Madrid, Las Ediciones de la Piqueta, 127-162.

Foucault, M. (2001) Los anormales. Curso del Collège de France (1974-1975). Madrid, Akal. Foucault, M. (2003) Hay que defender la sociedad. Curso del Collège de France (19751976). Madrid, Akal. 
Foucault, M. (2008) Seguridad, territorio y población. Curso del Collège de France (19771978). Madrid, Akal.

Foucault, M. (2009) Nacimiento de la biopolítica. Curso del Collège de France (1978-1979). Madrid, Akal.

Gallo, S. (2010) Filosofía, enseñanza y sociedad de control. Cuestiones de Filosofía, 12, 1-20.

García Fanlo, L. (2011) ¿Qué es un dispositivo?: Foucault, Deleuze y Agamben. A parte rei, 74, $1-8$

GrINBERG, S. (2008) Educación y poder en el siglo XXI. Gubernamentalidad y pedagogía en las sociedades de gerenciamiento. Buenos Aires: Miño y Dávila.

GrinberG, S. (2011) La conjetura del AND. Vitalpolitics y conducción de la conducta en la educación de nuestros tiempos, en Castro, E.; Grinberg, S.; O'Malley, P. y Veiga-Neto, A. (eds.) Biopolitica, gubernamentalidad, educación, seguridad. Buenos Aires, UnIPE, 13-24.

Hanushek, E. A. y Ludger, W. (2011) How much do educational outcomes matter in OECD countries? Economic Policy, 26, 427-491. http://dx.doi.org/10.1111/j.14680327.2011.00265.x.

HaRAWAY, D. J. (1995) Ciencia, cyborgs y mujeres. La reivindicación de la naturaleza. Valencia, Cátedra.

Hardt, M. y Negri, A. (2002) Imperio. Barcelona, Paidós.

JaKobi, A. P. y MARTENS, K. (2007) La influencia de la OCDE en la política nacional, en BONAL, X.; Tarabini-Castellani, A. y Verger, A. (eds.) Globalización y Educación. Textos Fundamentales. Buenos Aires, Miño y Dávila, 233-253.

Lazzarato, M. (2005) Biopolitique/Bioéconomie. Multitudes, 22 (automne), 1-7. http:// dx.doi.org/10.3917/mult.022.0051.

Lazzarato, M. (2009) Neoliberalism in action: inequality, insecurity and the reconstitution of the social. Theory, culture \& society, 26 (6), 109-133. http://dx.doi. org/10.1177/0263276409350283.

LuENGo, J. y SAURA, G. (2012) Mecanismos endógenos de privatización encubierta en la escuela pública. Políticas educativas de gestión de resultados y rendición de cuentas en Andalucía. Profesorado. Revista de Currículum y Formación del Profesorado, 16 (3), 111-126.

Luengo, J. y SAura, G. (2013) La performatividad en la educación. La construcción del nuevo docente y el nuevo gestor performativo. REICE. Revista Iberoamericana sobre Calidad, Eficacia y Cambio en Educación, 11 (3), 139-153.

LYOTARD, J.-F. (1991) La condición posmoderna. Informe sobre el saber. Madrid, Cátedra.

MARTENS, K. y ANJA, P. J. (2010) Introduction: The OECD as an Actor in International Politics, en Kerstin, M. y JaKobi, A. P. (eds.) Mechanisms of OECD Governance: International Incentives for National Policy-Making? Oxford, Oxford University Press, 1-25. http:// dx.doi.org/10.1093/acprof:oso/9780199591145.003.0001.

Masschelein, J. y Simons, M. (2002) An Adequate Education for a Globalized World? A note on the immunization of being-together. Journal of Philosophy of Education, 36, 565584. http://dx.doi.org/10.1111/1467-9752.00297.

Merchán, F. J. (2012) La introducción en España de la política educativa basada en la gestión empresarial de la escuela: el caso de Andalucía. Archivos Analíticos de Políticas Educativas, 20 (32), 1-28.

Meyer, H.-D. y Benavot, A. (2013) Introduction. PISA and the Globalization of Education Governance: some puzzles and problems, en Meyer, H. D. y Benavot, A. (eds.) PISA, 
Power and Policy. The emergence of a global educational governance. Oxford, Symposium Books, 9-26.

http://dx.doi.org/10.1093/acprof:osobl/9780199682331.003.0001.

http://dx.doi.org/10.1093/acprof:oso/9780199599332.003.0001.

http://dx.doi.org/10.5422/fordham/9780823253708.003.0012.

http://dx.doi.org/10.7208/chicago/9780226058009.003.0001.

http://dx.doi.org/10.1515/9781400823437.3.

http://dx.doi.org/10.9783/9780812207644.1.

http://dx.doi.org/10.5149/northcarolina/9781469610764.003.0001.

http://dx.doi.org/10.1515/9783110338478.1.

Moro, Ó. (2003) ¿Qué es un dispositivo? EMPIRIA: Revista de Metodología de las Ciencias Sociales, 6, 29-46.

Preciado, B. (2008) Testo yonqui. Madrid, Espasa-Calpe.

Preciado, B. (2011) Manifiesto contra-sexual. Prácticas subversivas de identidad sexual. Barcelona, Anagrama.

Rizvi, F. y Lingard, B. (2009) Globalizing educational policy. London, Routledge.

Robertson, S. L. y Dale, R. (2009) The World Bank, the ImF, and the Possibilities of Critical Education, en Apple, M. W.; Au, W. y Gandin, L. M. (eds.) The Routledge International Handbook of Critical Education. New York, Routledge, 23-35.

Rose, N. (2007) The Politics of Life Itself: Biomedicine, Power, and Subjectivity in the Twenty-First Century. Princeton, Princeton University Press. http://dx.doi. org/10.1515/9781400827503.

SAHLBERG, P. (2006) Education reform for raising economic competitiveness. Journal of Educational Change, 7, (4) 259-287. http://dx.doi.org/10.1007/s10833-005-4884-6.

Santos, M. (2013) Educación y construcción del Self en la Filosofía Helenística según Michel Foucault. Revista Española de Pedagogía, 256, 479-492.

Sellar, S. y Lingard, B. (2013) PISA and the Expanding Role of the OeCD in Global Educational Governance, en Meyer, H. D. y Benavot, A. (eds.) PISA, Power and Policy. The emergence of a global educational governance. Oxford, Symposium Books, 185-206.

Simons, M. (2006) Learning as Investment: notes on governmentality and biopolitics. Educational Philosophy and Theory, 38 (4), 523-540. http://dx.doi.org/10.1111/j.14695812.2006.00209.x.

Simons, M. y Masschelein, J. (2008) From Schools to Learning Environments: The Dark Side of Being Exceptional. Journal of Philosophy of Education, 42 (3-4), 687-704. http:// dx.doi.org/10.1111/j.1467-9752.2008.00641.x.

SloterdiJK, P. (2012) Has de cambiar tu vida. Sobre antropotécnica. Valencia, Pre-textos.

Stobart, G. (2008) Testing times: The uses and abuses of assessment. Abingdon, Routledge.

Tassin, E. (2012) De la subjetivación política. Althusser/Rancière/Foucault/Arendt/Deleuze. Revista de Estudios Sociales, 43, 36-49. http://dx.doi.org/10.7440/res43.2012.04.

Veiga-Neto, A. (2013) Biopolítica, normalización y educación, en Ruvituso, M. (ed.) Cuadernos de Pensamiento Biopolítico Latinoamericano/1 Buenos Aires, unipe, 10-19.

Verger, A. y AltinyelKen, H. K. (2012) Global education reforms and the new management of teachers: A critical introduction, en Verger, A.; Altinyelken, H. K. y Koning, M. D. (eds.) Global managerial education reforms and teachers: emerging policies, controversies and issues in developing contexts. Brussels, Education International, 1-18. 